\title{
Aplicación de imágenes NDVI para el control de riego y enfermedad en cultivos Agrícolas mediante el uso de aeronaves no tripuladas (UAV) y el software AgVault
}

\author{
Application of NDVI images for the control of irrigation and disease in \\ agricultural crops through the use of unmanned aircraft (UAV) and AgVault \\ software \\ Daniel Antonio Caballero Beltrán ${ }^{1}$
}

\section{RESUMEN}

El uso de tecnologías en agricultura se ha estado desarrollando intensamente. Por lo cual el uso de drones ya es actualmente aplicado. La agricultura de precisión es una herramienta necesaria hoy en día para poder ser eficiente y eficaz. Este estudio se realizó en la empresa American Color Inc. que está ubicada en el estado de Virginia - Estados Unidos. El objetivo es controlar el riego que este cultivo (Chrisante Mum) tiene; son 4.2 acres de área de cultivo y la técnica de riego utilizado es por goteo. Usando un Dron Phantom 3 DJI con cámara infrarroja Sentera (Single) identificamos posibles problemas y los corregimos. Mediante la aplicación del índice de vegetación NDVI y el software AgVault identificamos que sectores del cultivo necesitan más riego y otros solo control, este proceso interactúa con el campo y gabinete. El resultado es una colección de imágenes que al momento de interpretarlas y examinarlas podemos notar problemas y efectuar su remediación. En conclusión, se observa que el ahorro de agua y el control de enfermedades y/o plagas son problema; esto beneficia al ambiente por ser eficientes en los usos de químicos, también económicamente gracias al ahorro de agua y químicos.

Palabras clave: drones; NDVI; infrarrojo; índice de vegetación.

\section{ABSTRACT}

The use of innovative technologies in agriculture has been developing intensely; So the use of drones in agriculture is already applied. This study was conducted at the company American Color Inc. which is located in the state of Virginia, United States.

1 Universidad Nacional Agraria La Molina. Lima, Perú. 
The objective is to control the irrigation that this crop "Mum" has. Using a Dron Phantom 3 with infrared camera Sentera. With this we were able to calculate, through the application of the NDVI vegetation index and the AgVault software, which sectors of the crop need more irrigation and that others only control. The result is greater water savings and disease control, This not only benefits the environment; But, economically, it is profitable.

Keywords: drone; NDVI; infrared; vegetation index.

\section{INTRODUCCIÓN}

La tecnología en agricultura ha ido progresando enormemente, desde la maquinaria hasta los softwares, desde los bioquímicos hasta los orgánicos. La precisión en esta es necesaria ya que con ella podemos ser rentables y podremos solucionar varios problemas. La agricultura de precisión es una herramienta necesaria para obtener resultados reales pero esta necesita ser experimentada para poder ser usada. Es por ello que con el paso del tiempo los científicos, ingenieros y cultivadores han estado ideando máquinas, herramientas y otros para poder ser rentable en la agricultura. Los drones son un claro ejemplo, ya que estas aeronaves no tripuladas son usadas como máquinas recreativas, profesionales y militares; hay de diferentes formas y tamaños como también de precios y usos. Los drones para la agricultura están actualmente en auge: es por ello que en Estados Unidos existen una gran cantidad de empresas dedicadas a la agricultura de precisión que venden paquetes tecnológicos de drones especiales para agricultura. Estos drones tienen instalados en ellos cámaras que capturan fotos en infrarrojo, ya sea cercana o térmica, que tienen la capacidad de poseer imágenes de hasta 2 megapíxeles de resolución. Específicamente Sentera es una empresa dedicada a la venta de drones agrícolas que tiene un buen servicio y es de prestigio; la cámara térmica Single-NIR de Sentera es pequeña, ligera y precisa. Esta cámara capta la longitud de onda de infrarrojo cercano con el cual podemos calcular el NDVI. El índice de vegetación NDVI ayuda a entender la salud del cultivo, ya sea por riego o por enfermedad. Este índice se halla con la reflectancia de la luz solar y las hojas de las plantas. Es por ello que es una herramienta de decisión necesaria en agricultura. El NDVI es la combinación de imágenes en RGB y en infrarrojo en una simple ecuación. El resultado de estas imágenes nos ayuda a tomar decisiones de riego y aplicaciones, ya sea de fungicidas o insecticidas. En el Invernadero de American Color Inc. es necesaria la obtención de este índice para poder ser más precisos en la agricultura. American Color Inc. es una empresa dedicada a la horticultura. Está ubicada en el condado de Orange en el estado de Virginia en Estados Unidos de América. Es aquí donde se aplica esta tecnología y gracias a esta es uno de los mejores 100 horticultores de USA según la revista Greenhouse Grower`s, que reporta el top100 en invernaderos de Estados Unidos. El objetivo de esta investigación es controlar el riego y las enfermedades de los cultivos oportunamente con bajo costo. Siendo así eficaces, eficientes y amigables con el ambiente. La aplicación 
se hizo en cultivos de flores llamados Chrysanthe Mum que son plantas ornamentales con varios botones y de follaje verde oscuro.

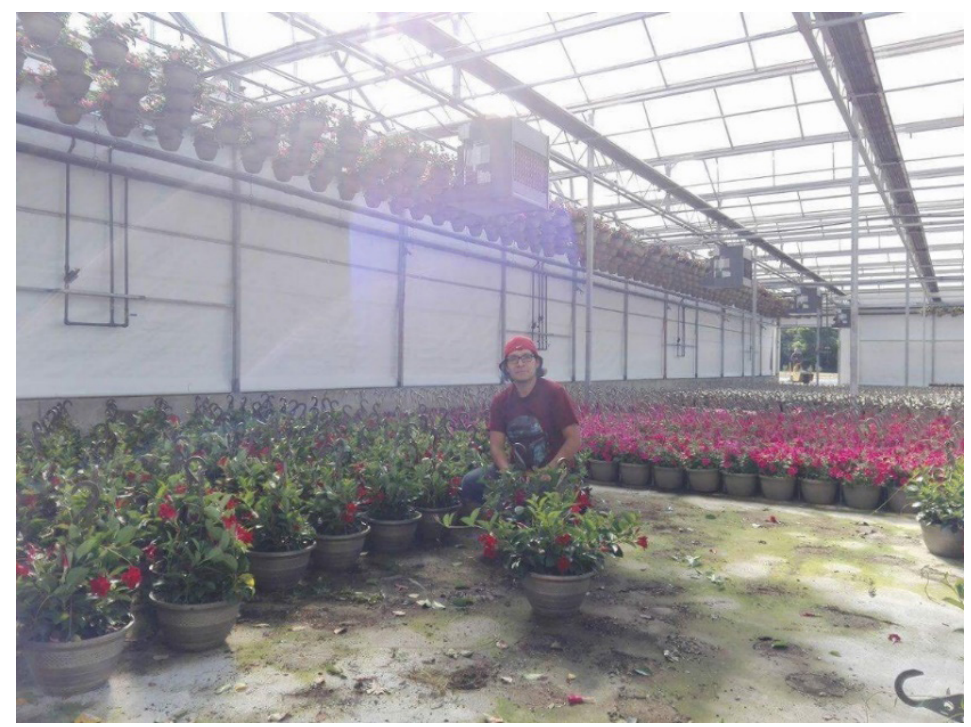

Figura 1. Cultivo de Clematis en American Color Inc.

\section{MATERIALES Y MÉTODOS}

Preparación y calibrado de la aeronave

Las aeronaves necesitan ser calibradas e identificar un plan de trabajo para poder ir al campo con todas las herramientas necesarias. El modelo Phantom 3 advance de la marca DJI es un dron profesional que es muy fácil de manejar. Antes de empezar a tomar las muestras en el campo es necesario calibrar este dron con el control y ajustarlo con la cámara Single-NIR de Sentera. Para ello se hace lo siguiente:

1) Calibrado del dron Phantom 3

Se instalan los propellers en el dron, dos de movimiento horario y dos de movimiento anti-horario. Estos son fáciles de identificar gracias a los colores que tienen. Luego, verificar que la batería del dron y del control remoto estén bien cargadas. Esto es necesario para poder iniciar el vuelo (DJI, 2016). Con la Aplicación DJI go para Phantom 3 o Matrice, descargada de la Apple store o google play, se inicia el calibrado; dando vueltas al dron vertical y horizontalmente, el control remoto reconoce las hélices y con esto se calibra el dron. La interacción entre el control y el dron debe ser precisa ya que de esta depende la seguridad del vuelo. 


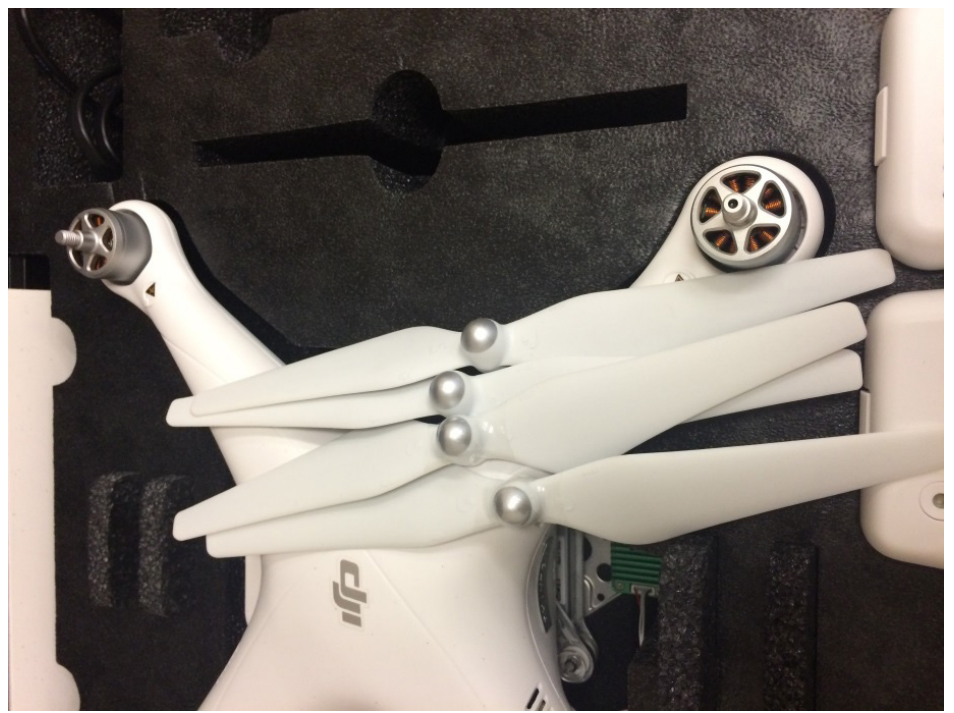

Figura 2. Propelles del Dron Phantom 3 Advance

2) Ajustar el dron con la cámara Single-NIR

La empresa Sentera ha desarrollado el software AgVault con el cual el dron puede interactuar con la cámara NIR. Este software tiene una aplicación para celulares, con ella el dron se ajusta con la cámara. Es necesario ser usuario activo de Sentera para poder tener la aplicación en un dispositivo, ya sea celular o tableta (Sentera, 2017). La compañía Sentera transforma el dron en una herramienta para la agricultura de precisión poniéndole la cámara NIR, todo este proceso es construido y desarrollado por ellos. Es así que el ajuste de la cámara con el dron es un proceso cerrado.

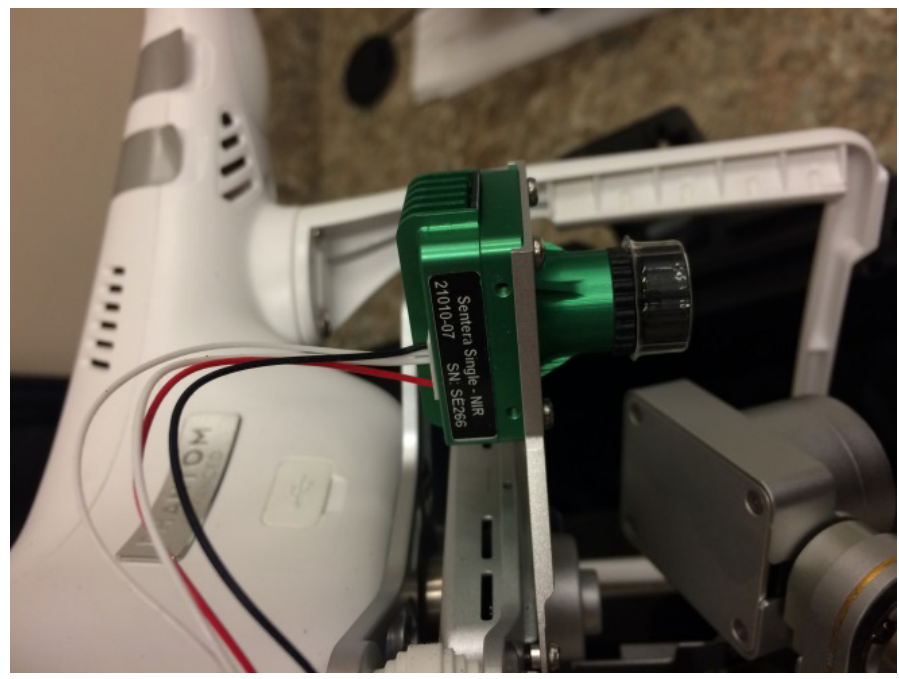

Figura 3. Cámara Single NIR de Sentera 
3) Plan de ejecución en el campo

Es necesario efectuar un plan para poder ir a recoger los datos al campo. Estos pueden ser: buena conexión de internet, equipos auxiliares de campo (estacas, sogas, etc), cálculo de altura de vuelo y direcciones de vuelo.

\section{Obtención de imágenes}

Para la obtención del índice NDVI es necesaria la adquisición de la información del cultivo. Esta información la obtenemos mediante imágenes. Son dos tipos de imágenes que se necesitan para crear el índice. Una es RGB que es de color verdadero, y la otra es la NIR infrarrojo cercano (Mahajan y Raj, 2017). Para ello se practica lo siguiente:

1) Planear el área

Es necesario calcular el área que se planea fotografiar. Esta tiene que ser un 2 a $3 \%$ más grande que el área del cultivo (Sentera, 2017). El planear el vuelo es esencial ya que las fotos deben ser unidas y por ello se necesita tener los puntos exactos para tomar las fotos. La cámara RGB tiene 12 megapíxeles de resolución y la NIR 1,2 megapíxeles.

2) Utilización de la aplicación AgVault

En campo es necesario ver que el dron pueda volar, luego de ello necesitamos abrir la aplicación de AgVault de Sentera. Esta aplicación está diseñada para tomar las fotos de ambas cámaras instantáneamente. Para ello solo se requiere establecer el área que necesitamos observar. Para personas con experiencia la altura de vuelo y vibración puede ser modificable. Entonces el dron empezará a dar su vuelo y aterrizará después de tomar todas las fotos. El tiempo de vuelo depende del área de observación. Las condiciones ideales de vuelo son: soleado, con vientos menos de $10 \mathrm{~m} / \mathrm{s}$ y establecer el área de despegue y aterrizaje mayor a 1,2 metros de diámetro.

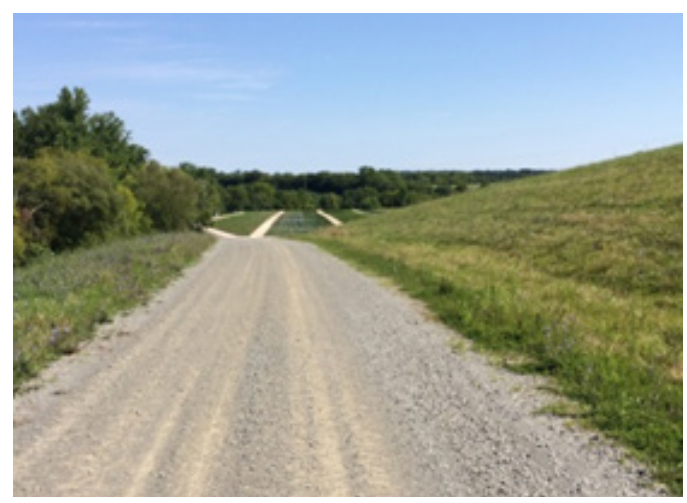

Figura 4. Área de estudio, cultivo Mum en D-Field 


\section{Construcción del NDVI y análisis}

El índice de vegetación es una ecuación que interactúa con las imágenes RGB y NIR.

Este índice se interpreta como el resultado de la reflectancia de la luz solar por parte de la clorofila en la planta. Clorofila absorbe luz RGB y refleja la luz infrarroja. Cuando la hoja está dañada o seca esta planta absorbe más luz infrarroja (Taipale, 2017 y William et al., 2017). Y gracias a ello podemos notar qué plantas están bien y qué plantas, no. El proceso es el siguiente:

1) Importación de imágenes

En gabinete la importación de imágenes es sencilla. Cada cámara tiene su memoria microSD con esta es fácil importarlas a la computadora. El software.

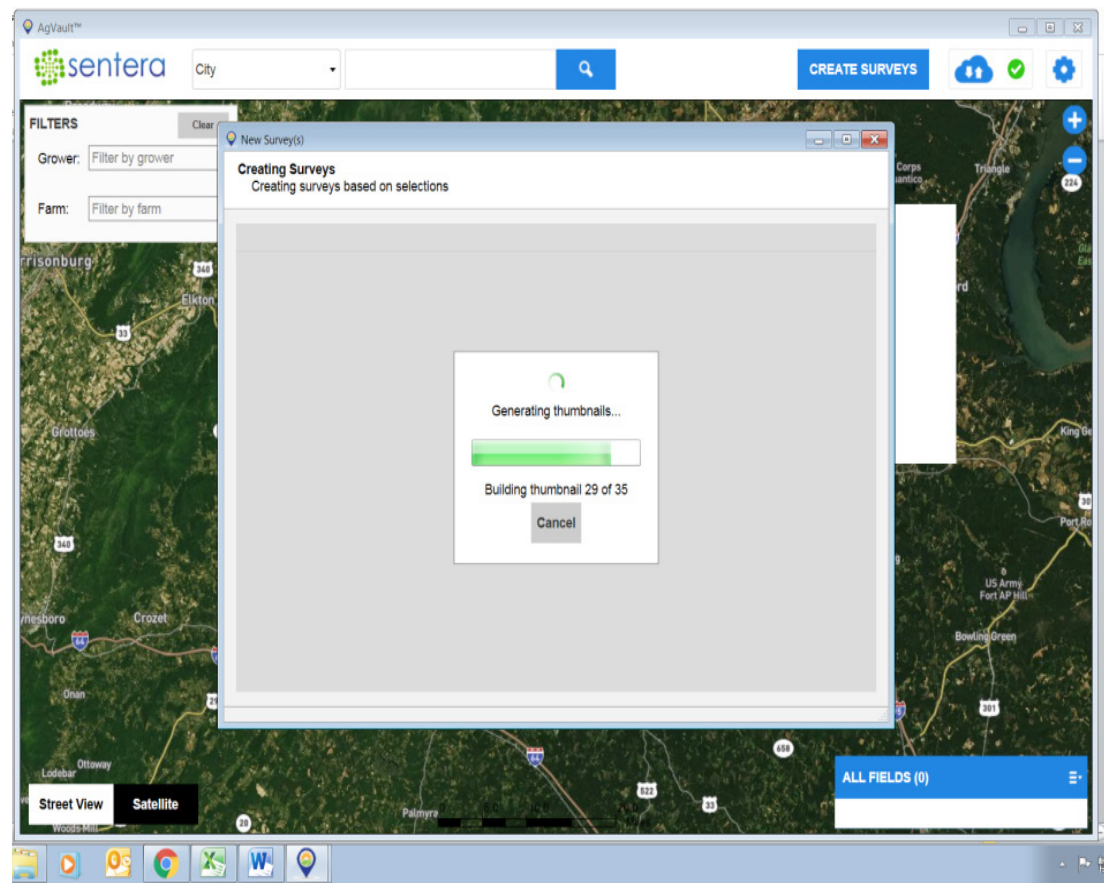

Figura 5. Contorno del programa AgVault en proceso

AgVault para escritorio es la herramienta que utilizaremos; este programa agrupa las fotos NIR y RGB de las cámaras. Las identifica fácilmente, solo es necesario precisar cuáles son de RGB (dron) y cuáles de NIR (Santera, 2017). 
2) Análisis del NDVI

El Programa procesa las imágenes RGB y NIR y las escala según los puntos tomados previamente.

Photo \& Boundary Confirmation

(a) imported. Adjust boundaries as needed.

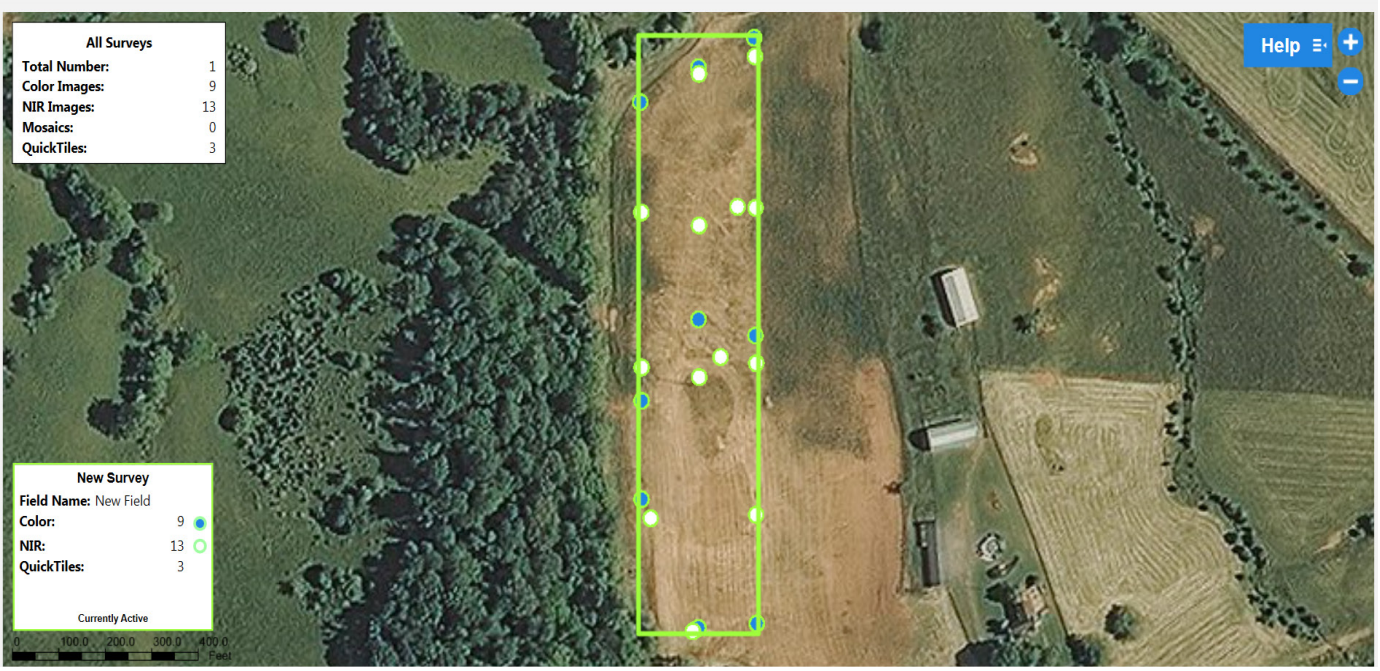

Figura 6. Puntos de procesamiento para AgVault

Luego de estas tenemos la imagen NDVI

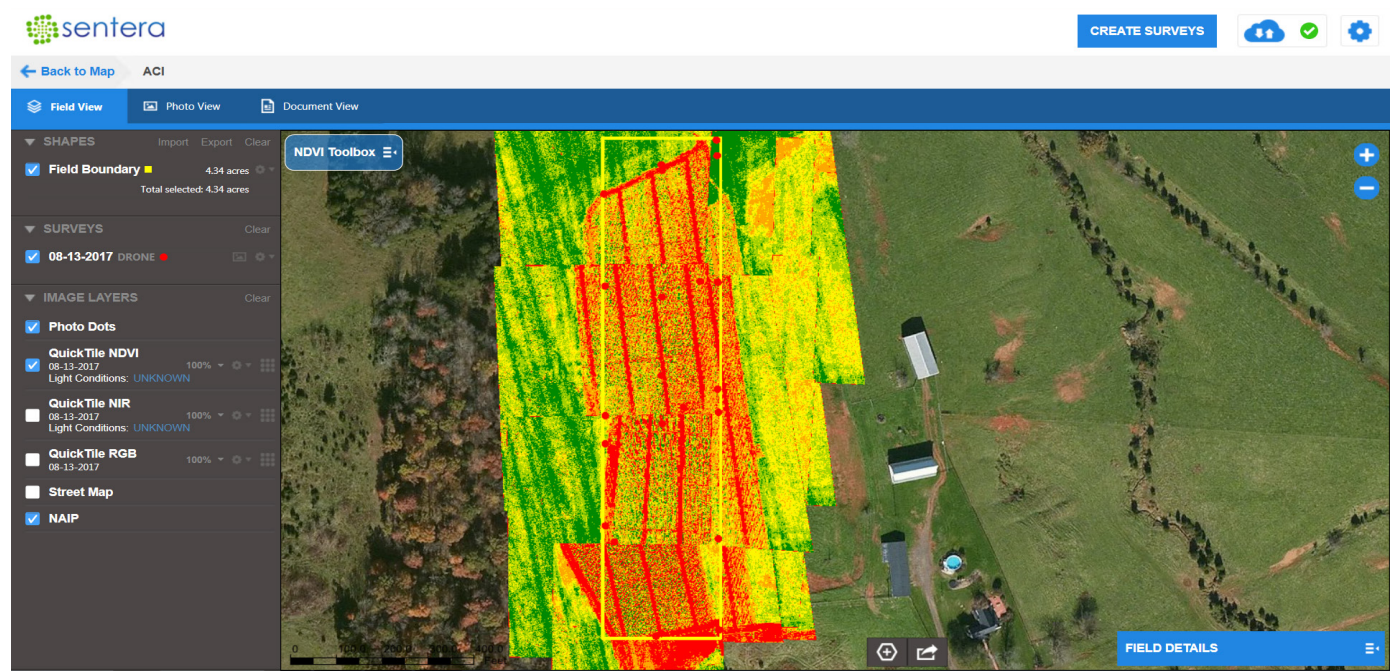

Figura 7. Resultado de procesamiento, imagen NDVI de todo el cultivo 


\section{RESULTADOS}

Una vez terminado todo el proceso por el cual obtenemos la imagen NDVI, necesario analizar e identificar los riesgos. Esta parte es muy importante ya que es fundamental para la toma de decisiones. Los siguientes fueron los resultados de esta toma de imágenes:

1) Se puede notar partes muy rojas, esto significa baja reflectancia de infrarrojo. Especialmente en la parte central del área del cultivo.

El resultado es contrastado en campo y con ello se toma la decisión. En esta parte del cultivo se determinó que las plantas estaban secas por un error técnico de los goteros. Se arregló el error de presión de los goteros y se corrigió el déficit.

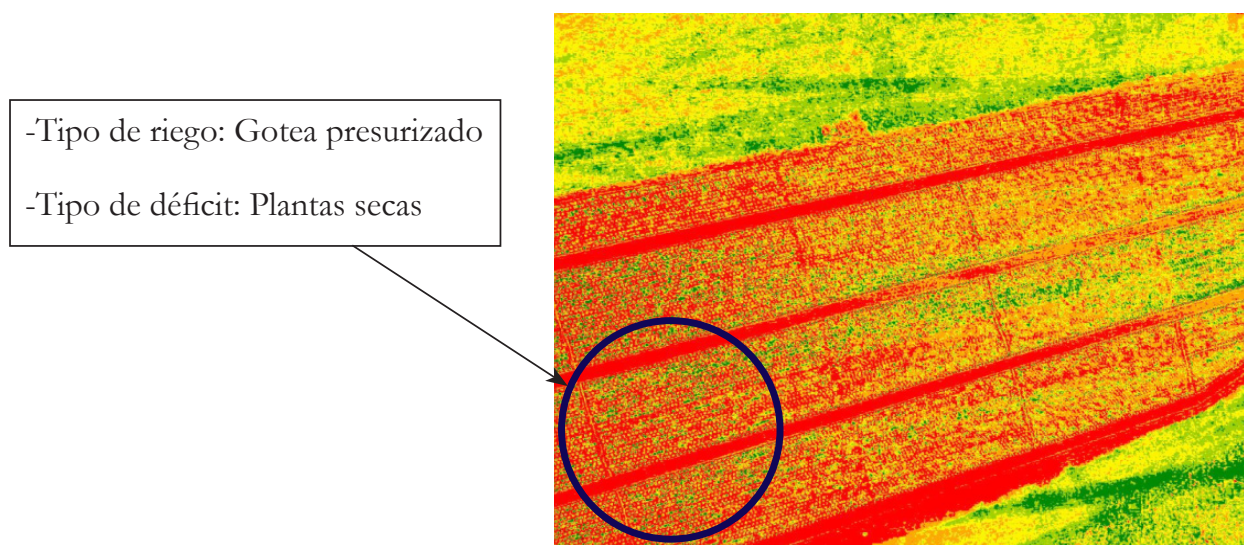

Figura 8. Análisis de imagen

2) Podemos comparar la imagen RGB, NIR y NDVI. Esto es necesario para poder calibrar la imagen NDVI en un futuro estudio. Esta comparación nos sirve como punto de partida para el siguiente estudio de campo y por lo tanto para datos históricos.

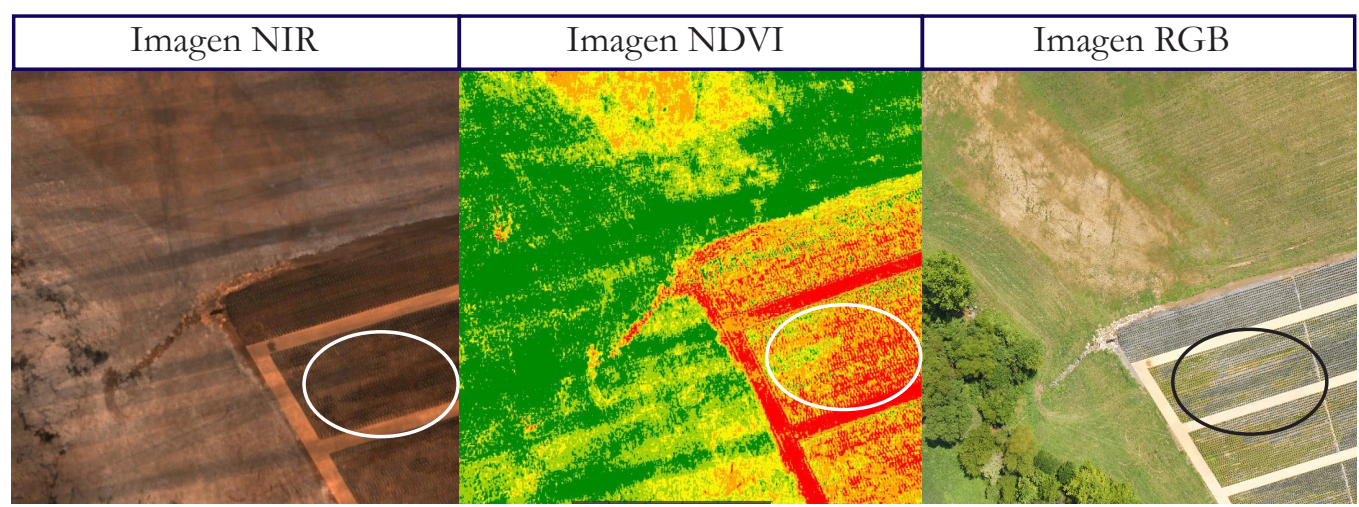

Figura 9. Comparación de imágenes 
3) Luego de notar algunas anomalías en los cultivos gracias a las imágenes, podemos tomar decisiones. Una de las decisiones más exactas es tomar una planta de control para poder analizarla en el laboratorio. Las muestras de laboratorio comunes que tomamos son: muestra de suelo (Media), muestra de hoja (Tessue) y muestra de ferti-riego (Water)

4) Cuando obtenemos plantas que están enfermas, la decisión es inmediata. La medida de control para proteger el cultivo es aplicar un químico para el problema. Normalmente el NDVI establece una conexión exacta con problemas de hongos en las hojas, por ello normalmente se aplica fungicida como Daconil Ultrex o Peagent.

\section{DISCUSIÓN}

1. Sobre el manejo del dron: es muy sencillo, lo más usual que pueda pasar es la pérdida de estabilidad debido a vientos fuertes o propelles rotos. Pero en general, es muy fácil manejarlos y acostumbrarse a ellos.

2. Sobre el manejo del software: el programa AgVault es muy sencillo y prácticamente hace todo el trabajo técnico, es rápido y los requisitos que necesita no son muy altos. Se podría decir que el mayor defecto de este es el uso de internet para interactuar con él. Pero en conclusión su uso es muy metódico te ahorra muchas horas de procesado.

3. Sobre el riego: después de ver el déficit en las imágenes, uno de los problemas más comunes son el riego. Este debido a problemas técnicos o cantidades pequeñas. Por lo tanto esta aplicación tecnológica ayuda en el riego tecnificado.

4. Sobre las muestras de laboratorio: luego de ver el resultado es muy importante, pero no necesario, enviar muestras de tierra, agua y hojas al laboratorio. Esto servirá para hacer la muestra y establecer puntos de control. Esta práctica incrementa la rentabilidad y disminuye el peligro de pérdida del cultivo.

\section{CONCLUSIONES}

Gracias a la agricultura de precisión con la herramienta NDVI, podemos aumentar nuestra rentabilidad y disminuir peligros para cualquier cultivo. Esto es un claro ejemplo del estudio realizado donde se demuestra que el monitoreo de cultivos con precisión es barato con los drones. En esta temporada los drones son herramientas muy útiles y no tan caros. Hay equipos desde 900 dólares hasta 9000 dólares pero la inversión se recupera a largo plazo porque los beneficios de esta herramienta son mayores. El índice de vegetación normalizado es ahora un recurso muy usado en la agricultura mundial, esto es por la simpleza del cálculo comparado con los beneficios obtenidos. 


\section{REFERENCIAS BIBLIOGRÁFICAS}

DJI, 2016. Quick start guide for Phantom 3 advanced. DJI documents and manuals $<$ https://dl.djicdn.com/downloads/phantom_3/20170706/Phantom+3+Professional+Release+Notes_.pdf > [Consulta: 25-07-2017].

Mahajan, Ujjwal y Raj, Bharat. 2017. Drones for Normalized Difference Vegetation Index (NDVI), to estimate Crop Health for Precision Agriculture: A Cheaper Alternative for Spatial Satellite Sensors. International Conference on Innovative Research in Agriculture, Food Science, Forestry, Horticulture, Aquaculture, Animal Sciences, Biodiversity, Ecological Sciences and Climate Change (AFHABEC-2016), At Jawaharlal Nehru University.

Sentera, 2017. Getting started and your first flight. Sentera movile guide <https://sentera. freshdesk.com/support/solutions/articles/19000055143-2-getting-started-with-your-first-import> [Consulta: 17-07-2017].

Taipale, Eric. 2017. NDVI and your farm: Understanding NDVI for plant health insights. $<$ https://sentera.com/what-is-ndvi-infographic/> [Consulta: 20-07-2107].

William John. et al. 2017. «Determination of green leaves density using normalized difference vegetation index via image processing of in-field drone - captured image». Journal of telecomunucation electronic and computer engineering. Vol. 9. 2-6

Recibido: $28 / 09 / 17$

Aceptado: 31/10/17

\section{Correspondencia}

Daniel Antonio Caballero Beltrán

daniel_caball@outlook.com 